can supply extremely useful information in the management of these patients. ${ }^{8}$

1 Karliner, J. S., Circulation, 1972, 46, 212.

2 Szidon, J. P., Pietra, G. G., and Fishman, A. P., New England fournal of Medicine, 1972, 286, 1200 .

${ }^{3} \mathrm{Katz}$, S., et al., American Review of Respiratory Disease, 1972, 106, 472.

4 American fournal of Medicine, 1971, 50,521.

Robin, E. D., Cross, C. E., and Zelis, R., New England fournal of Medicine, $1973,288,292$

6 Unger, K. M., Shibel, E. M., and Moser, K. M., Chest, 1975, 67, 8.

7 Connolly, D. C., Kirklin, J. W., and Wood, E. H., Circulation Research, $1954,2,434$.

8 Stevens, P. M., Friedmann, G. K., and Nictra, M. B., American Review of Respiratory Disease, 1973, 107, 1111.

\section{Epilepsy in the Elderly}

The onset of epilepsy in adult life is an important and familiar clinical problem, but discussion has usually centred on the relative merits of methods of detecting cerebral tumours with little attention to the practical management of the patients. This aspect assumes particular importance when epilepsy develops in the elderly, in whom the more rigorous neuroradiological investigations are inappropriate and in whom the hazards of craniotomy are increased by the infirmities of age. Hildick-Smith ${ }^{1}$ has emphasized recently that most published reports ${ }^{2-5}$ have been on patients seen in neurological centres, to which patients with the multiple disabilities commonly seen in geriatric hospitals are unlikely to be referred. In her own series of 50 geriatric patients with epilepsy of recent onset a potentially reversible cause was found in four: one had intermittent heartblock, one drug-induced hypoglycaemia, and two raised levels of blood urea. A cerebral tumour was found in five patients, four being metastatic and one primary; these diseases were not amenable to other than palliative treatment and survival was brief. The commonest cause was cerebrovascular disease culminating in hemiplegia, and another common clinical association was dementia of unknown cause. Clinicians are uneasily aware that both sudden hemiplegia and dementia are among the modes of presentation of meningioma, and assessment of the necessity and value of elaborate investigation remains a difficult problem.

Most tumours found to be the cause of epilepsy after the age of 60 are malignant, only one recent series containing any number of benign tumours. ${ }^{4}$ After the age of 70 tumours become increasingly uncommon as a cause of fits. ${ }^{5}$ In at least half the patients no cause can be found, there is no evidence of disease, and good control over the fits (often entirely nocturnal) can be established with anticonvulsants. The indications for investigation to discover a primary intracranial cause are not therefore very strong. However, an elderly patient whose health would justify craniotomy if a benign tumour should be found ought to be investigated by harmless means.

A high degree of success in finding brain tumours has been claimed for the combined use of the electroencephalograph and isotope scan. ${ }^{6} 7$ Both techniques will, however, also show abnormalities in patients with cerebral infarction, though with less tendency to persist. In fact the E.E.G. contains a high proportion of "abnormalities" in aged but otherwise apparently healthy individuals, ${ }^{8}$ and only a slow wave (delta) focus can be considered relevant. The combination of these techniques with radiography of the skull and echoencephalography has been claimed to detect all cerebral tumours ${ }^{9}$; but the many patients with false positives submitted unnecessarily to angiography would certainly be even greater in the elderly and is unacceptable.

In practical terms cardiac and metabolic causes must be excluded, and radiography of the chest and skull may show evidence of primary or secondary carcinoma, or of pineal shift, or raised intracranial pressure. The dorsum sellae is often poorly calcified in old age and this may be misleading. Lumbar puncture should not be done unless meningitis (infective or carcinomatous) is suspected. More elaborate investigation must largely depend on the individual patient. There can be no virtue in searching for brain tumours in patients over 80 or in obviously poor general health. Any patient who is otherwise well and has no evidence of brain disease or has symptoms and signs of a progressive focal lesion should certainly be investigated by both E.E.G. and scan. If there is clear evidence of a focal lesion thought not to be due to an infarct the decision on whether to proceed to angiography should be taken in consultation with the neurosurgeon. If no such focal lesion is shown further investigation is not required, and anticonvulsant treatment has a good chance of success.

In a patient who, on a reliable history, seems to have had a stroke and later develops fits it would be reasonable not to investigate further unless there is evidence of progressive disease. Both E.E.G. and scan will be abnormal, and there is nothing to suggest that serial investigation is more reliable than serial physical examination. The elderly patient with dementia and epilepsy is extremely difficult to investigate. Movement may prevent scanning, the E.E.G. will be very abnormal whatever the cause, and air encephalography is harmful. Such patients are most unlikely to harbour a remediable intracranial cause for their symptoms. No doubt all these uncertainties will be clarified when computerized transverse axial scanning (the E.M.I. scannner) ${ }^{10}$ is available in centres throughout the country.

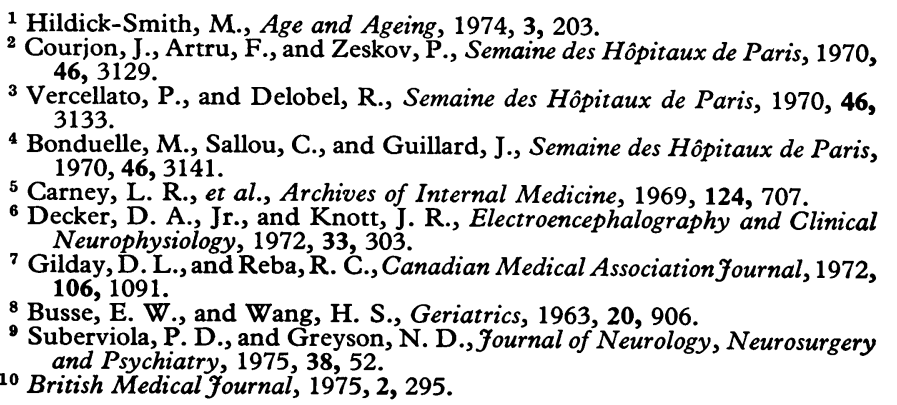

\section{Sodium Nitroprusside in Anaesthesia}

Induced hypotension during anaesthesia provides the surgeon with optimum operating conditions for such procedures as primary excision in massive burns, radical surgery of the head and neck, and the surgical treatment of phaeochromocytoma, cerebral aneurysms, and coarctation of the aorta. Profound hypotension is of great value in transsphenoidal hypophysectomy, dacrocystorhinostomy, and middle ear surgery. ${ }^{1}$ In the past few years interest has been reawakened in the use of sodium nitroprusside (SNP) as a hypotensive agent. It was first ${ }^{2}$ used clinically in 1929 and introduced into anaesthetic practice ${ }^{3}$ in 1962 , and since then has been used increasingly in anaesthesia and for the treatment of hypertensive encephalopathy. 